\title{
Planeación participativa en la expansión metropolitana de Caracas
}

\section{Participative Planning in the metropolitan expansion of Caracas}

\author{
Lacabana, M iguel y Cecilia Cariola (coords.) (2006), \\ ENTRE LA CIUDAD GLOBAL Y LA PERIFERIA EN TRANSICIÓN, CARACAS- \\ Valles del Tuy M edio, M inisterio del Poder Popular para Cien - \\ cia y Tén ología, Caracas, 107 Pp., ISBN : 978-980-6889-12-5
}

¿H acia dónde transita Caracas en esta etapa histórica de dominación neoliberal y profundos cambios sociales? ¿Puede pensarse que tiende a convertirse en una ciudad global? ¿La expansión metropolitana la convertirá en un supercentro que continuará absorbiendo a las poblaciones aledañas en su implacable dinámica de crecimiento concentrador?

Estas son las tres preguntas principales que guían la exposición y permiten vislumbrar un camino incierto que a la fecha preocupa y es motivo de diversos estudios, uno de los cuales a continuación se reseña.

En efecto, centrando el análisis en los complejos procesos de fragmentación y segmentación socioterritorial en Caracas, cuya expansión metropolitana ha incorporado ya a los valles del Tuy M edio, pero asimismo en las estrategias colectivas de las organizaciones de la sociedad civil (osc) y los cambios en las políticas territoriales del actual gobierno de la República Bolivariana, el M inisterio del Poder Popular editó en 2006 el texto colectivo coordinado por Cecilia Cariola y M iguel Lacaban intitulado Entrela ciudad global y la periferia en transición, Caracas-Valles del Tuy Medio, donde se exponen los resultados de investigación y un conjunto de interesantes reflexiones, debidamente fundamentadas en teorías y teorizaciones pertinentes para el caso de estudio.

El primer tema es la expansión metropolitana en el marco de la globalización, donde se establecen tres tendencias principales: a) la metropolización extendida, vinculada con el crecimiento de las ciudades como consecuencia de la producción posfordista y las nuevas actividades glo bal es, con un irrefrenable proceso de suburbanización; b) cambios en la economía urbana producto de la reestructuración de los mercados metropolitanos de traba- 
jo, con la dualidad laboral entre calificación y precariedad, y c) las transformaciones en la estructura interna de las ciudades como consecuencia de la agudización de los procesos de segregación residencial con creciente división socioespacial entre diversos segmentos urbanos, acompañada de mayor desigualdad. Aquí los planteamientos de Soja $(2000)^{1}$ resultan idóneos, así como la metáfora de múltiples ciudades, de los mismos autores del texto en cuestión, enfatizando la dimensión sociocultural de los procesos de cambio en aquella metrópolis. La idea de un proceso de globalización de Caracas, considerado en términos de la articulación de procesos globales y locales cierra el primer análisis, subrayando el impacto diferenciado sobre diversos grupos sociales, en buena medida opuestos sociopolíticamente, en una especial y conflictiva coyuntura histórica.

El segundo tema, también problemático, busca responder a la interrogante específica de si Caracas se encuentra en proceso de globalización o no. El punto es que, si bien la capital venezolana no se ubica en el centro de la economía global, su inserción en la jerarquía de ciudades globales tiene mucho que ver con el petróleo, lo que tempranamente implicó un carácter rentista de su economía, así como sus nexos con las compañías transnacionales petroleras, en una condición de subordinación a los circuitos existentes de la economía internacional. Sin embargo, con la nacionalización petrolera en 1976 cambió la situación y el gobierno tomó, hasta ahora, el control de ese importantísimo recurso para beneficio de todos los venezolanos. Con el Gran Viraje económico (1989-1992) y la consiguiente apertura petrolera, los tecnócratas a cargo de la empresa estatal Petróleos de Venezuela asumieron alegremente el discurso neoliberal, dando paso a una progresiva reprivatización de la industria petrolera nacional, lo que minaba el poder del Estado para controlar su propia industria, así como para impedir la caída de los ingresos fiscales derivados de la exportación petrolera. La contraparte del postulado petróleo e inserción global, que parecía imponerse, ha sido el impulso de una serie de prácticas de resistencia que impidieron la consolidación del modelo neoliberal y que tienen como eje el proyecto político del gobierno de H ugo Chávez, el cual propugna por un cambio de institucionalidad y de las formas de vinculación global a partir de la premisa petróleo y desarrollo nacional.

${ }^{1}$ Edward Soja, Posmetropolis. Critical Studies of Cities and Regions, Blackwell, Oxford, 2000. 
Entonces, acudimos a la oposición entre dos modelos y dos visiones de desarrollo que conllevan diferentes formas de inserción global. Así, a la pregunta de si tal posición del gobierno supone un retroceso en el proceso de globalización de Caracas, porque se encuentra ésta atrapada en un conflicto sociopolítico donde la contraposición de perspectivas resulta crucial, sólo cabe aceptar la complejidad de la situación; pero ponderando que el nuevo modelo supone asimismo una alternativa de incorporación a la economía global, el cual además de valorar las actividades económicas se orienta fundamentalmente al entorno regional de América Latina, planteando un desarrollo sociometropolitano basado en la cohesión y la justicia social, con prioridad hacia la mejora en las condiciones de vida de la población, más allá de cualquier concepción de la competitividad de la ciudad. Tales cambios en el modelo, al no haberse consolidado y logrado mayor justicia social y equidad, profundizaron los conflictos sociales y la fragmentación socioterritorial se ha hecho más compleja.

Enseguida el texto da cuenta de la expansión metropolitana de Caracas y la diferenciación de la periferia, aportando un conjunto de datos poblacionales y del mercado de vivienda, con base en los cuales ilustra la idea de la ciudad primaria y su interfaz periurbana o subregiones periféricas hacia donde la ciudad se expande y con la que mantiene una importante dinámica en cuanto a recursos, mercancías, energía y población, ubicando la década de los noventa como el periodo en que se consolidó la diferenciación, misma que se relaciona con la creciente desigualdad y la escasa presencia del Estado en la construcción de viviendas y la oferta privada dirigida a los sectores de mayores ingresos. Aparecen ahí los Valles del Tuy M edio (VTM ) como parte de la expansión de la periferia.

Como en muchos otros casos en América Latina, la expansión sobre los Valles del Tuy M edio tuvieron que ver con la prohibición de localizar nuevas industrias en Caracas y la obligación de trasladar aquellas empresas contaminantes, unido esto a la descentralización industrial a corta distancia y en un espacio delimitado. Es así que para los años ochenta el crecimiento económico impulsó el crecimiento demográfico, lo que a su vez atrajo importantes contingentes que llegaron a engrosar la población marginal, lo que derivó en una sobresegregación urbana como expresión de la concentración poblacional de la pobreza. Esto último tenía que ver con un cambio desde la periferia relegada a la pobreza de la periferia emergente con mayor heterogeneidad 
social. Las tendencias actuales son: 1) socioterritoriales: creciente urbanización de los VTM con diversificación socioterritorial interna, donde se acentua un eje más homogéneo de concentración de pobres, y otro más heterogéneo donde se ubican sectores de mayores ingresos; 2) tendencias socioambientales: falta de equilibrio entre los sistemas natural y urbano, presión sobre los recursos de agua, contaminación por aguas residuales y conflictos relacionados con ella; 3) tendencias socioeconómicas: fortalecimiento de Caracas como centro generador de empleo, intensificación de la terciarización de la economía local con predominio de las actividades informales, en tanto que la metrópolis concentra los servicios especializados vinculados a la economía global, así como una diversificación de la economía de la subregión, asimismo, resalta la inequidad de género como un rasgo dominante en la misma; 4) se registra un cambio de una región pobre a una con mayor inclusión social: se trata de un proceso que ha permitido a muchas familias incorporarse a los sectores que cuentan con acceso a la educación y la salud, lo que repercute en su poder adquisitivo y mayores oportunidades de salir adelante, y 5) las tendencias socioinstitucionales completan el cuadro, destacando el surgimiento de una institucionalidad subregional en los VTM que ha logrado articular a los gobiernos locales para emprender acciones integrales en el área, contribuyendo a potenciar el desarrollo local y la conformación de un centro submetropolitano que coadyuve a la transformación de la Región M etropolitana de C aracas como una metrópolis policéntrica. Todo lo anterior, acompañado de una tendencia a fortalecer la participación ciudadana mediante los mecanismos establecidos en la Constitución de 1999 y en las leyes sobre la institucionalidad local, por una parte, y por otra, nuevas formas de participación popular con una base territorial muy definida, que implica mayor o menor intervención del Estado.

Completan y dan perspectiva al análisis las respuestas sociales, como estrategias y modos de vida en la periferia. Aquí se considera la importante migración de sectores medios de Caracas hacia los VTM que acceden a viviendas construidas por el sector privado a un costo menor que las similares edificadas en el Área M etropolitana de Caracas (AM C), incluyendo la invasión y la llegada de sectores de damnificados de diversas áreas de Caracas que no contaban con vivienda. Así, las estrategias de vida son heterogéneas, justo como la población ahí radicada. Los sectores populares pobres, con acciones de carácter adaptativo, inmediatistas, replega- 
das al ámbito doméstico, frente al avance de la exclusión y la pobreza. Ante ello, las tendencias empiezan a cambiar, se dice, como consecuencia de las acciones gubernamentales incluyentes y participativas, sobre todo en materia de educación y salud, además de apoyo para la adquisición de suelo, infraestructura y saneamiento. Asimismo, aparecen propuestas populares de integración social y territorial, así como la esperanza en un futuro mejor. Ante la escasez de empleo y dificultades para costear los gastos el ementales, la población pobre no sólo acude al recurso del recorte en su consumo, sino también a la invasión de terreno, sin dejar de mantener y reforzar las redes sociales familiares, situación que ha implicado un encierro territorial que favorece la concentración de esfuerzos para sobrevivir en el plano doméstico. Este conjunto de esfuerzos, actualmente, encuentran un mejor cauce debido a la implantación efectiva y dinámica de la democracia participativa, que les permite intervenir directamente en los asuntos públicos, a la vez que revaloran y tienen cierta confianza en la acción gubernamental. Por su parte, los sectores medios han encontrado una opción de vivienda en la periferia, ciertamente con algunos costos para la vida cotidiana; pero en vista de haber sido excluidos del mercado de vivienda en el AM C, es en loS VTM donde han encontrado la opción de acceder a una vivienda propia; aunque los costos señalados tienen que ver con la posible pérdida del empleo, o bien por las distancias y gastos extra que tienen que solventar. No obstante, las estrategias de reproducción son clave, en la medida que permiten y conllevan una reorganización de la cotidianidad con base en el empleo. En realidad, las estrategias de este sector son similares a las de las clases populares, si bien manteniendo una condición de vulnerabilidad asociada a la precariedad del empleo, la lejanía y la difícil comunicación con los centros de trabajo y los altos costos del transporte. A pesar de ello, el balance es favorable debido a la mejoría de la vivienda, la incorporación extensiva de jóvenes a la educación superior y la viabilidad de las actividades informales que desarrollan. Se trata, finalmente, del surgimiento de estrategias colectivas y cambios en el modo de vida en la periferia metropolitana que implican nuevas formas de participación popular, impulsadas desde las políticas públicas, con un fuerte anclaje territorial, donde continúan surgiendo las organizaciones y las redes solidarias que favorecen la participación y movilización de las comunidades identificadas territorialmente a fin de dar respuesta a las necesidades colectivas, mejorando la calidad de vida, a la vez que crean espacios para la construcción y ejercicio de la ciudada- 
nía responsable. Puede afirmarse que la gente construye comunidad, accede a las instituciones y, sin negar las tensiones propias de los conflictos sociopolíticos, avanza en la organización popular, fortaleciendo la vida barrial y preservando sus derechos y potencialidades.

Como ejemplo y a la vez corolario del encuentro entre acciones colectivas y políticas públicas orientadas a la justicia social, se cuenta con un marco institucional que ha generado una nueva institucionalidad local en la periferia metropolitana. Esto se ilustra con el caso de los Consejos Locales de Planificación Pública, que buscan la integración de las comunidades organizadas y grupos vecinales vía la participación y el protagonismo dentro de una política de Estado, contraponiéndose a la divisa neoliberal de más mercado menos Estado, en un caso de la mayor importancia como las M esas Técnicas de Agua. Aquí han surgido proyectos de cogestión entre el gobierno y organizaciones sociales, respetando los valores propios de la ciudadanía, como la tolerancia, la tenacidad, la responsabilidad, el encuentro de saberes técnicos y populares y el aprendizaje de derechos y compromisos, donde la comunidad tiene el papel protagónico, así como el proceso educativo, que a pesar de que las desconfianzas iniciales y la fal ta de iniciativa quedaron rebasados, se superaron las tensiones y dejaron aflorar respuestas sociales, lo que ha permitido el surgimiento de otras instancias organizativas, como los comités de tierras urbanas, de salud, cooperativas y otras que también se orientan a la participación ciudadana.

La respuesta final está en manos de las comunidades y la voluntad de transformar su realidad, acompañados siempre, en mi opinión, de un gobierno comprometido y leal.

Recibida: 14 de enero de 2008. Aceptada: 28 de enero de 2008.

José M aría Aranda-Sán chez Universidad Autónoma del Estado de M éxico Correo-e: arandas_301@ hotmail.com

José María Arandaránchez. Es licenciado en psicología (UN AM), maestro en sociología (UAEM) y doctor en urbanismo (UNAM). ACtualmente es profesor-investigador de tiempo completo en el Centro de Investigación en Ciencias Sociales y H umanidades de 
la Universidad Autónoma del Estado de M éxico (cıcsyn). Sus líneas de investigación son: movimientos sociales en M éxico y organizaciones de la sociedad civil. Es líder del cuerpo académico: Investigacion en ciencias sociales y humanidades, del сісsyн, integrante del comité editorial de la revista Contribuciones desde Coatepec y miembro del Sistema $\mathrm{N}$ acional de Investigadores, nivel I. Entre sus publicaciones recientes se encuentran: "R edes sociales y reflexividad: su importancia en la construcción de la sociedad civil en M éxico", Ciencia Ergo Sum, 14 (2), UAEM, M éxico, pp. 141-150 (2007); "Perspectiva de género para el análisis de la participación femenina en organizaciones ambientalistas: el caso de la organización de mujeres ecologistas de la sierra de Petatlán, Gro.", Territorios, 16-17, Universidad del Rosario, CoIombia, pp. 107-125 (2007). 